\title{
A importância da associação entre o cuidado humanizado e intervencionista na sala parto: uma revisão de literatura
}

The importance of the association between humanized and interventional care in the delivery room: a literature review

La importancia de la asociación entre atención humanizada e intervencionista en la sala de partos: revisión de la literatura

Matheus de Almeida Schittini ${ }^{1 *}$, Gyovanna Rodrigues Cardoso², Yasmin Cavalcanti Braga ${ }^{3}$, Kaliane Alcantara Ouriques ${ }^{3}$, Laura Frinhani Valadão ${ }^{4}$, Gabriela Rezende de Oliveira ${ }^{5}$, Laura de Alcântara Matera $^{6}$, Isabelle Thomaz de Campos ${ }^{7}$, Mônica Isaura Corrêa ${ }^{8}$.

\section{RESUMO}

Objetivo: Revisar traçando um paralelo entre o cuidado humanizado e intensivo em neonatos com os benefícios dessas práticas na redução de intercorrências neonatais. Revisão Bibliográfica: Como a transição para a vida extrauterina envolve muitas mudanças fisiológicas no recém-nascido, faz-se necessário que os cuidados humanizados sejam cada vez mais aplicados nas salas de parto, como o contato pele a pele por meio do método Canguru e o aleitamento materno logo após o nascimento. Porém, precisa ser tomada algumas medidas profiláticas durante todo o processo de internação com intuito de impedir ou diminuir o início e desenvolvimento de patologias, podendo ser feitas por meio de triagem de deficiências congênitas, o cuidado para doenças com transmissão vertical e condutas profiláticas como a administração de vitamina $\mathrm{K}$. Considerações finais: Já se sabe a importância dos cuidados nas primeiras horas de vida do recémnascidos, mas que é necessário investir mais em cuidados humanizados para que a transição para a vida extrauterina seja a melhor pro RN, sem causar traumas a ele e, concomitantemente, sem deixar de lado os cuidados essenciais para avaliar que a saúde está bem.

Palavras-chave: Recém-nascido, Sala de parto, Assistência.

\begin{abstract}
Objective: Review by drawing a parallel between humanized and intensive care in neonates and the benefits of these practices in reducing neonatal complications. Bibliographic Review: As the transition to extrauterine life involves many physiological changes in newborns, it is necessary that humanized care be increasingly applied in delivery rooms, such as skin-to-skin contact through the Kangaroo method and breastfeeding soon after birth. However, some prophylactic measures need to be taken during the entire hospitalization process in order to prevent or reduce the onset and development of pathologies, which can be done by screening for congenital disabilities, care for vertically transmitted diseases, and prophylactic conducts such as the
\end{abstract}

\footnotetext{
${ }^{1}$ Centro Universitário do Espírito Santo (UNESC), Colatina - ES.

*E-mail: matheus almeida222@hotmail.com

2 Universidade Federal de Uberlândia (UFU), Uberlândia - MG.

${ }_{3}^{3}$ Universidade de Ciências Sociais Aplicadas (UNIFACISA), Paraíba - PB.

${ }^{4}$ Centro Universitário de Belo Horizonte (UNIBH), Belo Horizonte - BH.

${ }^{5}$ Faculdade de Minas (FAMINAS), Belo Horizonte - BH.

${ }^{6}$ Universidade José do Rosário Vellano (UNIFENAS), Belo Horizonte - BH.

7 Universidade de Cuiabá (UNIC), Cuiabá - MT.

8 Instituto Metropolitano do Vale do Aço (FAMEVAÇO), Ipatinga - MG.
} 
administration of vitamin K. Final considerations: The importance of care in the first hours of life of newborns is already known, but that it is necessary to invest more in humanized care so that the transition to extrauterine life is the best for the newborn, without causing trauma to him and, concomitantly, without leaving aside the essential care to assess that health is well.

Key words: Newborn, Delivery room, Assistance.

\section{RESUMEN}

Objetivo: Revisar trazando un paralelismo entre los cuidados humanizados e intensivos en neonatos y los beneficios de estas prácticas en la reducción de las complicaciones neonatales. Revisión bibliográfica: Dado que la transición a la vida extrauterina implica muchos cambios fisiológicos en los recién nacidos, es necesario que se apliquen cada vez más cuidados humanizados en las salas de partos, como el contacto piel con piel mediante el método canguro y la lactancia materna poco después del nacimiento. Sin embargo, es necesario tomar algunas medidas profilácticas a lo largo del proceso de hospitalización para prevenir o reducir la aparición y el desarrollo de patologías, lo que puede hacerse mediante el cribado de discapacidades congénitas, la atención de enfermedades con transmisión vertical y conductas profilácticas como la administración de vitamina K. Consideraciones finales: La importancia de los cuidados en las primeras horas de vida de los recién nacidos ya es conocida, pero que es necesario invertir más en cuidados humanizados para que la transición a la vida extrauterina sea la mejor pro RN, sin causarle traumas y, concomitantemente, sin dejar de lado los cuidados esenciales para evaluar que la salud esté bien.

Palabras clave: Recién nacido, Sala de partos, Asistencia.

\section{INTRODUÇÃO}

O passar dos anos trouxe mudanças nas mais diversas esferas científicas, com o processo de partejar não haveria como ser diferente, dessa forma houve modificações também na assistência ao Recém-Nascido (RN). Os primeiros relatos histórico-científicos acerca da concepção descrevem um processo primitivo, porém de grande enlace afetivo e corporal entre todos os envolvidos. Com a modernização da medicina surgiu a necessidade constante de uma assistência acelerada, haja vista a urgência de uma rápida desocupação de leitos obstétricos (AYRES LFA, et al., 2021).

Dessa maneira, foram priorizadas intervenções imediatas, muitas vezes desnecessárias e de caráter iatrogênico, especialmente em prematuros internados em unidades de terapia intensiva neonatal, que recebem um tratamento ostensivo e agressivo (KUGELMAN AMD, et al., 2018). Ainda que em neonatos que nasçam com baixo peso exista a necessidade de um tratamento mais enérgico, na maioria dos grandes centros hospitalares, a aspiração de vias aéreas, secção do cordão umbilical, profilaxia ocular, são priorizadas em detrimento da assistência humanizada, ainda que não exista nenhuma condição patológica que exija tais medidas (AYRES LFA, et al., 2021).

A mortalidade infantil no Brasil tem como responsável por cerca de $70 \%$ dos óbitos as causas relacionadas com intercorrências durante o período neonatal. Com a intenção de diminuir esse componente nas taxas de mortalidade, a assistência imediata ao RN no âmbito nacional sofreu modificações para garantir boas práticas de suporte perinatal em conformidade com a Rede Cegonha (AYRES LFA, et al., 2021). Elaborado pela Sociedade Brasileira de Pediatria, o Programa de Reanimação Neonatal, também contribui para que seja estabelecido um padrão de conduta no momento do nascimento. Tal programa tem credibilidade em todo o âmbito nacional e reforça a ideia de que intervenções antes rotineiras, como por exemplo a aspiração de vias aéreas, não devem ser realizadas. Ademais, fortalece as ideias previstas nas orientações da Rede Cegonha (GOMES MASM, et al., 2021).

A primeira hora pós-parto é fator determinante para a sobrevida no primeiro ano de vida do neonato. Neste período de tempo é recomendado que haja uma visão holística dos principais personagens atrelados a concepção. O contato pele a pele precoce e contínuo deve ser realizado com o bebê em decúbito ventral, 
sobre o tórax materno, ambos desnudos. Tal prática fortalece o vínculo entre a mãe e bebê, liberando ocitocina e prolactina, o que aumenta o desejo da parturiente de amamentar e regula a temperatura do $\mathrm{RN}$, o que diminui o risco de hipotermia, sendo a temperatura com parâmetro de normalidade entre $36,5-37,5^{\circ} \mathrm{C}$ retais (LEDO BC, et al., 2021). Além disso, esse ato de humanização repercute nas esferas fisiológicas e preserva o equilíbrio acidobásico, bem como ajuda a colonização bacteriana pela microbiota cutânea materna (AYRES LFA, et al., 2021).

Entretanto, para que isso ocorra de forma segura, deve-se realizar uma avaliação clínica do RN, com intuito de identificar se o neonato será admitido em alojamento ou será encaminhado a centros de terapia intensiva neonatal. É importante averiguar se a idade gestacional é maior ou igual a 35 semanas, bem como avaliar o tônus muscular e o padrão respiratório do RN. Caso todas as questões possuam respostas afirmativas recomenda-se que seja admitido em um berçário neonatal, mas que antes disso seja apresentado ao corpo materno, promovendo assim um estreitamento do vínculo mãe-bebê. Além disso, o início precoce e instruído da amamentação é importante e os bebês devem ser alimentados assim que possível para evitar condições hipoglicemiantes (MCLEE-GARRET TM, 2021).

As primeiras 6 horas após o parto são conhecidas como o período de transição entre a vida intra e extrauterina, sendo um intervalo de tempo marcado por intensas alterações fisiológicas. Tais mudanças requerem uma monitorização intensa e periódica, sendo repetidas a cada 30-60 min, para a avaliação dos cuidados e adaptação homeostática de seu corpo a um novo ambiente. Os principais parâmetros clínicos analisados são: a temperatura, haja vista que padrões de hipertermia e hipotermia persistentes podem levar à suspeição de sepse, a Frequência Cardíaca (FC) e a Frequência Respiratória (FR) que devem ser avaliadas durante os primeiros segundos de vida. Caso o neonato apresente-se taqui ou bradipneico, com FR menor que 40 Incursões Respiratórias Por Minuto (IRPM) ou maior que 60 IRPM, o RN deve receber suporte ventilatório nos próximos 30 segundos (MARSHALL S, et al., 2019).

Entre outras condições a serem avaliadas estão o tônus muscular e a coloração da pele, sendo a cianose indicativa de doença respiratória ou cardíaca (MARSHALL S, et al., 2019). Neste período deve ser administrado Vitamina K (fitonadiona), a fim de prevenir a Doença Hemorrágica do Recém-Nascido (HDN), bem como a vacinação contra Hepatite B. Além disso, é importante que seja realizado um cuidado asséptico após o clampeamento e secção do cordão umbilical e a triagem neonatal para avaliação de deficiências auditivas de caráter congênito (MCLEE-GARRET TM, 2021).

Diante do exposto, o presente trabalho objetivo traçar um paralelo entre o cuidado humanizado e intensivo em neonatos, buscando correlacionar o benefício de associar tais práticas na redução de intercorrências neonatais, bem como analisar as repercussões dessa forma de cuidado nos padrões metabólicos e no desenvolvimento neuropsicomotor durante os primeiros dias de vida do $\mathrm{RN}$.

\section{REVISÃO BIBLIOGRÁFICA}

\section{Benefícios da humanização e da Amamentação}

Durante as últimas décadas, a implementação de estratégias humanizadas no cuidado ao RN, na sala de parto, tornou-se foco de diversos estudos, buscando a redução de condutas desnecessárias após o nascimento. Muitos médicos ainda priorizam a realização de aspiração gástrica ou profilaxia ocular, no primeiro momento de vida do bebê, o que pode ser justificado pela ausência de protocolos institucionais ou alta demanda de serviços, com a necessidade de liberação da sala de parto (JUNG SM, et al., 2020).

A transição para a vida extrauterina envolve diversas alterações fisiológicas no RN, inclusive no padrão de trocas gasosas, equilíbrio ácido básico e atividade cardiovascular (LEDO BC, et al., 2021). Assim, torna-se clara a importância dos cuidados humanizados na sala de parto como: a presença de um acompanhante, ambiência, sonoridade, luminosidade, temperatura ambiente, clampeamento do cordão umbilical, prevenção da perda de calor do RN, CPP e amamentação (AYRES LFA, et al., 2021).

Assim, visando a melhoria do primeiro contato entre a mãe e o RN, políticas públicas de saúde, como o Método Canguru e a Iniciativa Hospital Amigo da Criança (IHAC), foram estabelecidas. O Método Canguru, 
criado em 1978, é pautado na atenção neonatal, preconizando o Contato Pele a Pele (CPP) entre a mãe e o bebê logo na primeira hora de vida, adotando a posição canguru que consiste em colocar o RN despido em decúbito ventral, entre os seios da puérpera. Já a IHAC, lançada em 1991, incentiva a prática do aleitamento materno baseada nos "10 passos para o aleitamento materno" e possui como um dos critérios, o auxílio às gestantes no início da amamentação na primeira meia hora após o parto (LAMOUNIER JA, et al., 2019).

O contato pele a pele, realizado na primeira hora de vida do RN, na posição canguru, é responsável por gerar diversos benefícios para o bebê como: a regulação da temperatura, a manutenção dos níveis sanguíneos de glicose, adaptação metabólica e manejo da dor (BRASIL, 2017). Porém, como analisado em estudo feito em uma maternidade na Zona da Mata Mineira, apenas $13,3 \%$ das gestantes que realizaram cesárea tiveram contato com o bebê indicando, assim, que a via de parto ainda tem forte influência sobre 0 CPP (AYRES LFA, et al., 2021).

Outra prática essencial na primeira hora de vida é o aleitamento materno, que tem elevado fator de proteção contra a morte neonatal e gera benefícios para a mãe, devido a liberação de ocitocina, estimulada pelo toque, calor e odor, promovendo contrações uterinas responsáveis por reduzirem a incidência de hemorragia pós-parto (JUNG SM, et al., 2020). Ademais, o leite materno possui componentes que reduzem infecções, diarreias e a hipoglicemia, além de a amamentação ser o momento no qual o vínculo entre a puérpera e o bebê é estabelecido, fortalecendo os laços afetivos entre eles.

Ainda foi comprovado, em estudo feito, que RN colocados em contato com o seio materno, tem 4,5 vezes mais chances de irem para o Alojamento Conjunto acompanhados da mãe, após a saída da sala de parto (LEDO BC, et al., 2021). Dessa forma, fica claro que métodos humanizados e a amamentação no primeiro momento são essenciais, devendo ser priorizados em detrimento a exames e outros procedimentos, que devem ser realizados posteriormente. Logo, a gestante deve ser informada sobre essas práticas, como 0 CPP, durante o pré-natal, a fim de gerar um primeiro momento benéfico tanto para ela quanto para o bebê (AYRES LFA, et al., 2021).

\section{Profilaxias necessárias durante a internação}

Ações profiláticas consistem em um conjunto de medidas, as quais visam impedir ou diminuir o início e desenvolvimento de determinada patologia. Neste ínterim, é de suma importância considerar os aspectos fisiológicos, ainda imaturos, dos neonatos como fatores de risco e de susceptibilidade a infecções oportunistas ou até mesmo transmissões verticais após o parto. Assim, uma das intervenções necessárias na garantia do bem-estar e para evolução do recém-nascido é a administração da vitamina $\mathrm{K}$, no intuito de prevenir doenças hemorrágicas, uma vez que essa está associada à formação da protrombina e também atua como um cofator essencial à enzima carboxilase influenciando os resíduos do ácido glutâmico na conversão das proteínas associadas à cascata proteolítica (MAJID A, et al., 2019).

Entretanto, a vitamina $\mathrm{K}$ não atravessa facilmente a placenta e sua concentração no plasma fetal é muito baixa. Junto a isto, a microbiota intestinal dos neonatos é inábil na produção da vitamina $\mathrm{K}$ e o aleitamento materno não contém os percentuais satisfatórios. Dessa forma, é orientado pela Sociedade Brasileira de Pediatria a administração via intramuscular ou oral da vitamina $\mathrm{K}$ sob o esquema: Via intramuscular 0,5 a 1 $\mathrm{mg}$ em dose única ou via oral, em duas doses, reposição na forma de fitomenadiona $1 \mathrm{mg}$ semanal até 12 semanas ou $2 \mathrm{mg}$ com 1, 7 e 30 dias de vida (MAJID A, et al., 2019).

Ademais, complementando a conduta de profilaxias aos neonatos, o método de Credè, aplicação do colírio de nitrato de prata a $1 \%$ nos olhos do recém-nascido, foi o tratamento primordial na prevenção à conjuntivite neonatal por transmissão vertical. Contudo, como é demonstrado no estudo Paiva ML, et al. (2018), inúmeros países substituíram o uso de nitrato de prata pela eritromicina $5 \%$ ou pomada de tetraciclina a $1 \%$ devido a maior eficácia desses na prevenção à clamídia e devido a análise das suscetíveis manifestações de conjuntivite química como consequência do uso do nitrato de prata. Diante disso, e considerando a epidemiologia brasileira, a Sociedade Brasileira de Pediatria reconheceu também a toxicidade do nitrato de prata e recomenda o uso desse somente na indisponibilidade do colírio povidona a 2,5\% ou da pomada de eritromicina a 0,5\% ou da tetraciclina a 1\% (MALIK A, et al., 2017). 
Por fim, sendo evidente a imunização como meio para redução da mortalidade infantil, a administração das vacinas Bacille Calmette-Guérin (BCG), para hepatite $B(\mathrm{HBV})$ e contra poliomielite [vacina oral contra poliomielite (OPV); ou vacina inativada contra poliomielite (IPV)] evita e diminui o risco dessas infecções nos primórdios de vida e é inerente à conduta profilática do neonato. Ademais, o Estatuto da Criança e do Adolescente (ECA) estabelece que é dever da família assegurar a efetivação dos direitos à saúde, o que inclui a vacinação do neonatal (WHITTAKER E, et al., 2018)

\section{Implantação de testes de triagem de deficiências congênitas}

O teste da orelhinha consiste em um exame que avalia o funcionamento da audição de recém-nascidos e também os possíveis futuros problemas auditivos que o bebê pode vir a ter. É válido ressaltar que o teste é indolor e realizado nos primeiros momentos de vida. No Brasil, quando é detectada alguma anomalia pelo teste disponibilizado na rede pública de saúde, o paciente é encaminhado para profissionais da área, para melhor resolução do quadro. O teste é de extrema importância para o desenvolvimento da comunicação, já que a plena capacidade auditiva está intimamente relacionada à produção da fala. Dessa forma, é indispensável a ampla implementação desse teste, tendo em vista que a anomalia representa, segundo estudos, a terceira deficiência mais comum no país e melhora consideravelmente a qualidade de vida (SANTOS E, et al., 2018).

O Teste do olhinho, também conhecido como Teste do Reflexo Vermelho, da mesma forma, detecta inúmeras patologias indispensáveis de serem detectadas com certa antecedência, a exemplo da leucocoria, identificada por um reflexo branco, diferente do usual (vermelho), podendo indicar, por exemplo, casos de catarata congênita e retinopatia da prematuridade. Ademais, podem ser descobertos, a partir do exame, glaucoma congênito, infecções oculares e ptose. Quando identificadas previamente, algumas dessas patologias podem ser tratadas fornecendo um maior grau de desenvolvimento para a criança (MONTENEGRO L, et al., 2018).

O teste do pezinho permite a identificação de doenças como hipotireoidismo congênito, fenilcetonúria, fibrose cística, doença falciforme e outras hemoglobinopatias, deficiência de biotinidase e hiperplasia adrenal congênita. $O$ ideal é que o teste seja realizado em até 30 dias após o nascimento, através de uma picada no calcanhar do recém-nascido, realizando uma pequena coleta de sangue. Disponível pelo Programa Nacional de Triagem Neonatal do Ministério da Saúde, o teste é obrigatório e fundamental para reduzir os índices de mortalidade, aumentar a qualidade de vida do neonato, além de reduzir futuros custos para o cofre público, por ser viável financeiramente e reduzir maiores gastos com decorrências das anomalias (FREITAS B, et al., 2015).

O teste do coraçãozinho, igualmente fornecido pelo do Sistema Único de Saúde e realizado por meio da aferição de oximetria de pulso, é um teste da triagem neonatal importante para o diagnóstico de cardiopatias congênitas. Essas patologias representam um elevado número de mortalidade infantil. Cerca de 2 em cada 1000 nascidos-vivos são portadores de cardiopatia congênita crítica e retratam aproximadamente $10 \%$ dos óbitos em crianças, podendo levar também a complicações como hipóxia e choque. Logo, a efetivação do teste é imprescindível e reduz consideravelmente os índices de mortalidade (QUEIROZ I, 2019).

\section{Doenças de transmissão vertical}

É importante analisar a relação entre o acompanhamento pré-natal da gestante e a transmissão vertical de algumas doenças, por exemplo, a transmitida pelo vírus da Imunodeficiência Humana (HIV), pelo vírus da hepatite B e C e pelo Treponema pallidum, causador da sífilis (FABIO SV, 2017). Além disso, doenças como rubéola, toxoplasmose, citomegalovírus e herpes simples também podem ser transmitidas verticalmente. A partir disso, será dado enfoque especial ao contágio por sífilis e por toxoplasmose (MORAES CL, et al., 2020).

A sífilis gestacional amplia a possibilidade de transmissão vertical, a qual, em casos não tratados, amplia em aproximadamente $40 \%$ o número de repercussões negativas, seja abortos espontâneos ou sequelas perinatais. Nesse cenário, ressalta-se a importância da implantação adequada do pré-natal, visto que é imprescindível na minimização de riscos e no diagnóstico adequado da grávida e de seu parceiro, por exemplo, por exames sorológicos (MACÊDO VC, et al., 2020). No caso da toxoplasmose, as manifestações 
clínicas na gestante são semelhantes a quadros gripais e estão associadas a algumas complicações, como afecções no Sistema Nervoso Central, no RN. Existem divergências quanto ao teste sorológico de rotina e o tratamento somente deve ser proposto nos casos de gestantes com toxoplasmose aguda, visto que não existe comprovação sobre a real eficácia do tratamento da fase aguda com a prevenção da transmissão para o feto (BARTHOLO BBGR, 2017).

\section{CONSIDERAÇÕES FINAIS}

Muito já se sabe a respeito dos cuidados necessários que são tomados nas primeiras horas de vida do recém-nascido a fim de diminuir a mortalidade por intercorrências. Para isso, primeiramente, deve-se fazer uma avaliação clínica do RN, e determinar a ida para o alojamento ou centros de terapia neonatal. O primeiro contato com a mãe após o parto traz inúmeros benefícios para o RN, tal como o aleitamento materno, que possui componentes que diminuem infecção, diarreia e hipoglicemia, além de ajudar no vínculo entre a mãe e o bebê. Outras práticas são adquiridas que visam diminuir intercorrências, como administração de vitamina $\mathrm{K}$, vacinas adequadas para a idade, e também testes de triagem de deficiências congênitas, como teste do pezinho, da orelhinha, olhinho e coraçãozinho. Além disso, deve-se sempre se atentar para o risco de doenças de transmissão vertical. Dessa forma, todas as transformações que ao longo do tempo foram criadas, tem o intuito de melhorar a assistência às gestantes e aos recém-nascidos.

\section{REFERÊNCIAS}

1. AYRES LFA, et al. Fatores associados ao contato pele a pele imediato em uma maternidade. Escola Anna Nery, 2021; 25: 2.

2. BARTHOLO BBGR. Avaliação da transmissão vertical da toxoplasmose em gestantes com infecção aguda no HUPE/UERJ. Dissertação (Mestrado em Ciências Médicas) - Universidade do Estado do Rio de Janeiro, Rio de Janeiro, 2017, $68 \mathrm{p}$.

3. BRASIL. Ministério da Saúde. Secretaria de atenção à saúde. Atenção humanizada ao Recém-Nascido. Brasília, 2017.

4. DOMINGUES RMSM, LEAL MC. Incidência de sífilis congênita e fatores associados à transmissão vertical da sífilis: dados do estudo Nascer no Brasil. Cadernos de Saúde Pública, 2016; 32: 1-10.

5. FABIO SV. Pré-natal do parceiro como estratégia para redução da transmissão vertical das doenças sexualmente transmissíveis e melhora dos indicadores de saúde perinatal. Dissertação (Mestrado em Ginecologia e Obstetrícia) Departamento de Ginecologia e Obstetrícia. Faculdade de Medicina de Ribeirão Preto da Universidade de São Paulo, Ribeirão Preto, 2016, 94p.

6. FREITAS B, et al. A importância do teste do pezinho. Revista Saberes, 2015; 3: 2-13.

7. GOMES MASM, et al. Atenção hospitalar ao recém-nascido saudável no Brasil: estamos avançando na garantia das boas práticas?. Ciência \& Saúde Coletiva, 2021; 26: 859-874.

8. JUNG SM, et al. Contato pele a pele: experiências de puérperas. Revista de Enfermagem do Centro-Oeste Mineiro, 2020; 10.

9. KUGELMAN AMD. Less is more: modern neonatology. Rambam Maimonides Medical Journal, $2018 ; 9: 11$.

10. LAMOUNIER JA, et al. Iniciativa hospital amigo da criança: 25 anos de experiência no Brasil. Rev. Paul Pediatria, 2019;37(4).

11. LEEDO BC, et al. Fatores associados às práticas assistenciais ao recém-nascido na sala de parto. Escola Anna Nery, 2021; 25: 10.

12. MACÊDO VC, et al. Sífilis na gestação: barreiras na assistência pré-natal para o controle da transmissão vertical. Cadernos de Saúde Coletiva, 2020; 28: 518-528.

13. MAJID A, et al. Profilaxia com vitamina K para recém-nascidos: uma perspectiva histórica para compreender as barreiras modernas de absorção. Hospital Pediatrics, 2019 9(1).

14. MARSHALL S, et al. Delivery room handling of the newborn. J.Pirimant Medical, 2019; $16: 10$.

15. MCLEE-GARRET TM. Overview of the routine management of the healthy newborn infant. Uptodate, 2021.

16. MIHATSCH WA, et al. Prevention of Vitamin K Deficiency Bleeding in Newborn Infants: A Position Paper by the ESPGHAN Committee on Nutrition. JPGN, 2016; 63(1).

17. MONTENEGRO L, et al. Importância do Teste do Olhinho. Semana de pesquisa da UNIT, 2018.

18. MORAES CL, et al. Infecção congênita - diagnóstico e tratamento materno-fetal. Research, Society and Development., 2020; 9(8): 1-32.

19. QUEIROZ I. A importância do teste do coraçãozinho no diagnóstico precoce de cardiopatias congênitas. Trabalho de Conclusão de Curso (Bacharelado em Enfermagem) - Centro Universitário do Planalto Central Apparecido dos Santos. Brasília, 2019.

20. SAKALA IG, et al. A eficácia da vacina neonatal e o papel dos adjuvantes, Expert Review of Clinical Immunology, 2019.

21. SANTOS E, et al. Teste da orelhinha. XVI Jornada Científica dos Campos Gerais. Ponta Grossa, 2018.

22. WHITTAKER E, et al. Imunização neonatal: Justificativa, Estado atual e perspectivas futuras. Frontiers in Immunology, 2018; 6. 